\title{
On the primary model to explain the relation between a rigidity-dependent spectral hardening of proton and helium spectra and a sharp knee of the all-particle spectrum
}

\author{
Ying Zhang ${ }^{1}$,J. Huang ${ }^{1}$, D.Chen ${ }^{2}$, H.B.Jin ${ }^{2}$, M. Shibata ${ }^{3}$, Y. Katayose ${ }^{3}$, L. M. Zhai ${ }^{1,2}$, \\ Xu Chen ${ }^{1}$, X.B. Hu ${ }^{1,4}$ and Y.H.Lin ${ }^{1}$ \\ ${ }^{1}$ Key Laboratory of Particle Astrophysics, Institute of High Energy Physics, Chinese Academy of \\ Sciences, Beijing 100049, China \\ ${ }^{2}$ National Astronomical Observatories, Chinese Academy of Sciences, Beijing 100012, China \\ ${ }^{3}$ Faculty of Engineering, Yokohama National University, Yokohama 240-8501, Japan \\ ${ }^{4}$ Department of Physics, Shandong University, Jinan 250100, China
}

E-mail: Vingzhangemail.ihep.ac.cn

\begin{abstract}
Cosmic Ray (CR) spectrum in some energy region can not be well expressed by a simple powerlaw as it has a structure. Recently, AMS-02, PAMELA, ATIC-2 and CREAM-2 presented a rigidity dependent CR spectra with a remarkable hardening in excess of around $300 \mathrm{GV}$. On the other hand, the all-particle energy spectrum of primary CRs observed in a wide range from $10^{14}$ $\mathrm{eV}$ to $10^{17} \mathrm{eV}$ with the Tibet-III AS array clearly shows a sharp knee at around $4 \mathrm{PeV}$. Based on these results, in this paper, we propose a phenomenological model capable of well explaining both the data by AMS-02 and others in the lower energy region and that by the Tibet AS $\gamma$ experiment simultaneously. We discuss some details of our model in which extra nearby CR sources are responsible for creating a sharp knee. This model also predicts a dominance of $\mathrm{CR}$ nuclei heavier than helium at the knee energies.
\end{abstract}

The 34th International Cosmic Ray Conference,

30 July- 6 August, 2015

The Hague, The Netherlands 


\section{Introduction}

The spectrum of CRs has offered a few clues to its origin so far. The only features observed are at very high and ultrahigh energies, the so-called knee at a few times $10^{15} \mathrm{eV}$, the second "knee" at $\sim 10^{18} \mathrm{eV}$, the "ankle" at higher energies, and a spectral steepening above $10^{18} \mathrm{eV}$ [四][四][目] [四]. But, recently CR measurements by the new-generation experiments such as the AMS-02,ATIC2, CREAM-2 and PAMELA seem to indicate that the CR spectrum deviates from a single power law in the rigidity region lower than $1 \mathrm{TeV}[[\mathrm{[}][\mathrm{G}][\mathrm{Z}][\mathrm{[}]$. These measurements have caused a wide range of interest. Many models were proposed to account for the hardening of the flux based on different sources, acceleration mechanisms, diffusive propagation effects, and their superposition [9]. On the other hand, The Tibet AS $\gamma$ Collaboration has measured the all-particle spectrum in a wide energy range between $10^{14} \mathrm{eV}$ and $10^{17} \mathrm{eV}$ by air-shower array of the area of $37000 \mathrm{~m}^{2}$ located at 4300m above sea level (Tibet, Yangbajing, China, atmospheric depth of $606 \mathrm{~g} / \mathrm{cm}^{2}$ ) and provided the most detailed spectrum around the knee which clearly shows a sharp knee at around 4 $\mathrm{PeV}[\mathrm{WU}]$. The origin of the knee in the energy spectrum of cosmic rays is an outstanding problem in astroparticle physics. Proposals for its origin range from astrophysical scenarios such as a change of acceleration mechanisms at the sources of cosmic rays (supernova remnants, pulsars, etc.), to a single-source assumption or effects due to propagation inside the galaxy (diffusion, drift, escape from the Galaxy), to particle physics models such as the interaction with relic neutrinos during

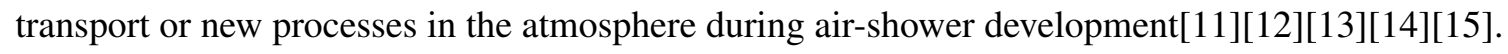
In order to resolve the origin of the knee, the detailed study of the chemical composition provides a key information since the change of the energy spectrum must be closely related to its chemical composition.

Based on these results, we here propose a phenomenological model capable of well explaining both the data by AMS-02 and others in the lower energy region and that by the Tibet AS $\gamma$ experiment simultaneously. We discuss some details of our model show that extra nearby CR sources can well explain a sharp knee. This model also predicts a dominance of cosmic-ray nuclei heavier than helium at the knee.

\section{Direct measurements and hardening spectrum}

Measurements of the energy spectra of different nuclei show a consistent concave shape over the common range 30-1000 GeV/nucleon (virtually the same rigidity) from Helium to Iron. Protons show a similar pattern up to a higher energy per nucleon. Appropriate approximation for the energy spectra of primary nuclei taking into account the rate of change of spectral slope was reported in [16] where $\mathrm{E}$ is the energy of a primary nucleus $\mathrm{A}$ with charge $\mathrm{Z}, \mathrm{R}=E_{k} / \mathrm{Z}$ is the rigidity dependent energy at which the asymptotic energy spectral power index $\gamma 1$ for $\mathrm{E}$ « $E_{k}$ is changed to the asymptotic value $\gamma_{2}\left(\gamma_{2}=\gamma 1+\Delta \gamma 1\right)$ for $\mathrm{E} » E_{k}$ at sharpness parameter $\xi$ correlating with the rate of change of the spectral slope. So the broken power-law energy spectrum can be expressed as

$$
h(E)=\Phi_{z} * E^{-\gamma 1} *\left(1+\left(E / E_{k}\right)^{s}\right)^{(-\Delta \gamma 1 / s)}
$$

Above about $10 \mathrm{GeV}$, the solar modulation is negligible. The reason for $\gamma 1$ being different for proton and other nuclei (heavier than proton) is beyond the scope of the present work, and 


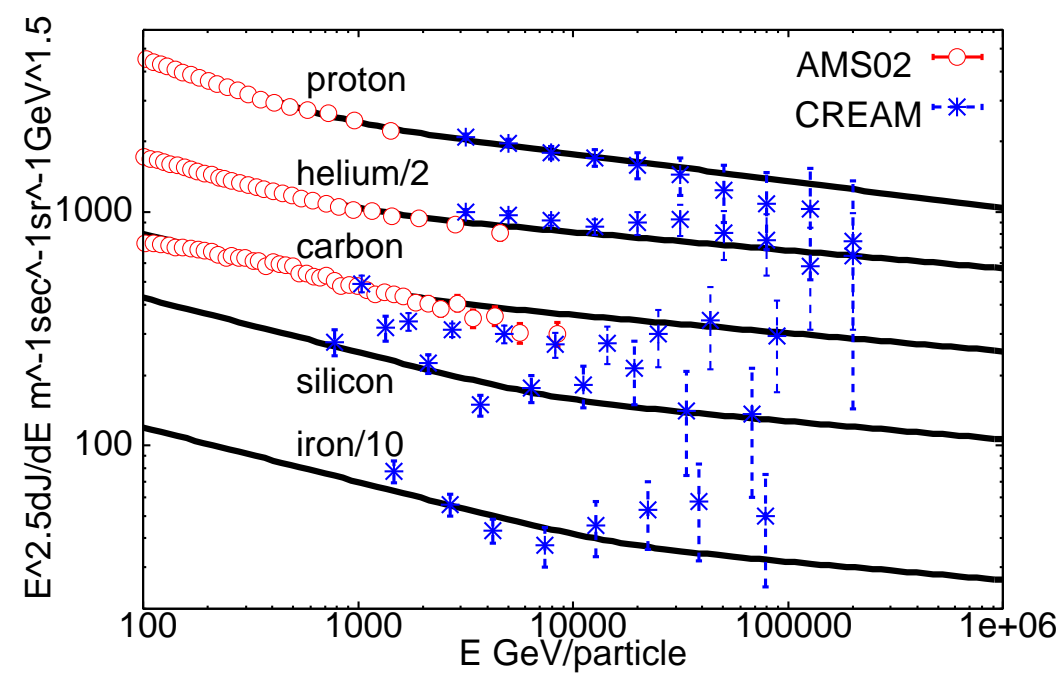

Figure 1: Elemental spectrum expressed by a broken power-law function at lower energy region $(<100$

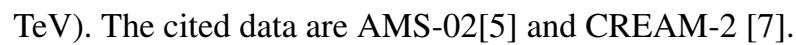

Table 1: Parameters of the Chemical Composition Used in the Present Work. The adopted values in function(2.1) for Spectra index $\gamma 1$, hardening energy $E_{k}$ and spectral index difference $\Delta \gamma 1$ are used to describe hardening spectrum at low energy, another two parameters: cutoff energy $E_{c}$ and spectral index difference $\Delta \gamma 2$ are obtained by fitting the function (3.1) to characterise the knee structure at high energy.

\begin{tabular}{|c|c|c|c|c|c|}
\hline nuclei & $\gamma 1$ & $E_{k}$ & $\triangle \gamma 1$ & $E_{c}$ & $\triangle \gamma 2$ \\
\hline $\mathrm{p}$ & 2.8 & $700 \mathrm{GeV}$ & 0.2 & $200 \mathrm{TeV}$ & 0.3 \\
\hline other & 2.7 & $5 * \mathrm{Z} \mathrm{TeV}$ & 0.16 & $200 * \mathrm{Z} \mathrm{TeV}$ & 0.3 \\
\hline
\end{tabular}

we divide proton and other nuclei as two groups. The values of $E_{k}, \gamma 1$ and $\Delta \gamma 1$ are taken from experimental data in the energy range from several $10^{10} \mathrm{eV}$ to several $10^{14} \mathrm{eV}$ obtained by AMS02 and CREAM-2. The adopted values for $E_{k}, \gamma 1$ and $\Delta \gamma 1$ are listed in Table 1, these parameters can be used to describe hardening spectrum at lower energy region $(<100 \mathrm{TeV})$. Our model use another two parameters: cutoff energy $E_{c}$ and spectral index difference $\Delta \gamma 2$ to characterise the knee structure at higher energy region $(>100 \mathrm{TeV})$ which can be seen below. Some experimental results of the direct observation for individual elements (proton, helium, carbon, oxygen, neon, magnesium, silicon and iron) are summarized in Fig. 1, in which the solid line represents a broken power-law function.

\section{Indirect experiments}

Individual spectra of primary CRs as directly measured at the top of the atmosphere for energies below $100 \mathrm{TeV}$, are extrapolated to high energies and fitted to results from air-shower measurements. We require this model should not only can well explain the data by AMS-02 and others in the lower energy region, but also simultaneously coinside to the all-particle spectrum measured by the Tibet AS $\gamma$ experiment which is the highest statistical and the best systematics-controlled measurement covering the widest energy range around the knee energy region. The energy spectra of 

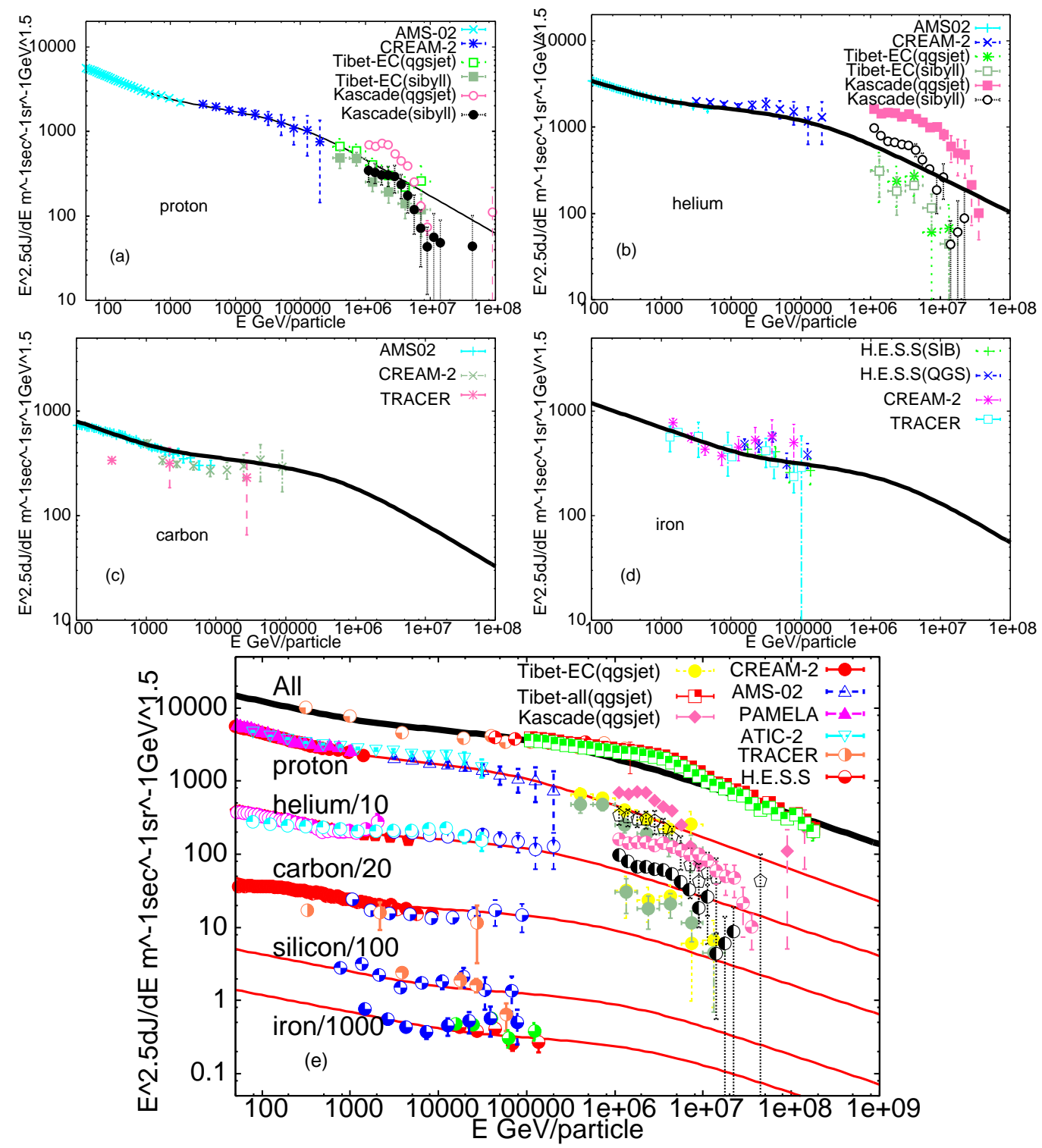

Figure 2: Energy spectra of proton(a), helium(b), carbon(c),iron(d) and the all-particle(e).References of the

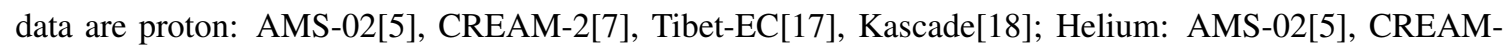

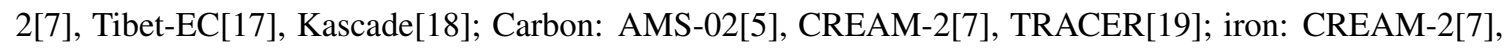

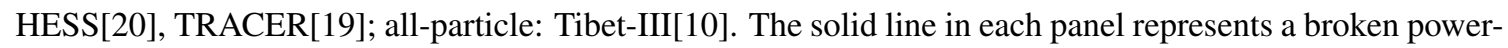
law type behavior of the energy spectra.

individual nuclei derived from extensive air shower experiments can also be described by a broken power-law function with a cut-off at high energies. We use this kind of function to parameterize the observed differential energy spectra for individual elements of CRs, which can be written as

$$
f(E)=h(E) *\left(1+\left(E / E_{c}\right)^{s}\right)^{(-\Delta \gamma 2 / s)}
$$

According to the Diffusive Shock Acceleration model, which is considered to be the most promising model for the acceleration of the galactic cosmic rays at SNRs, the acceleration limit 


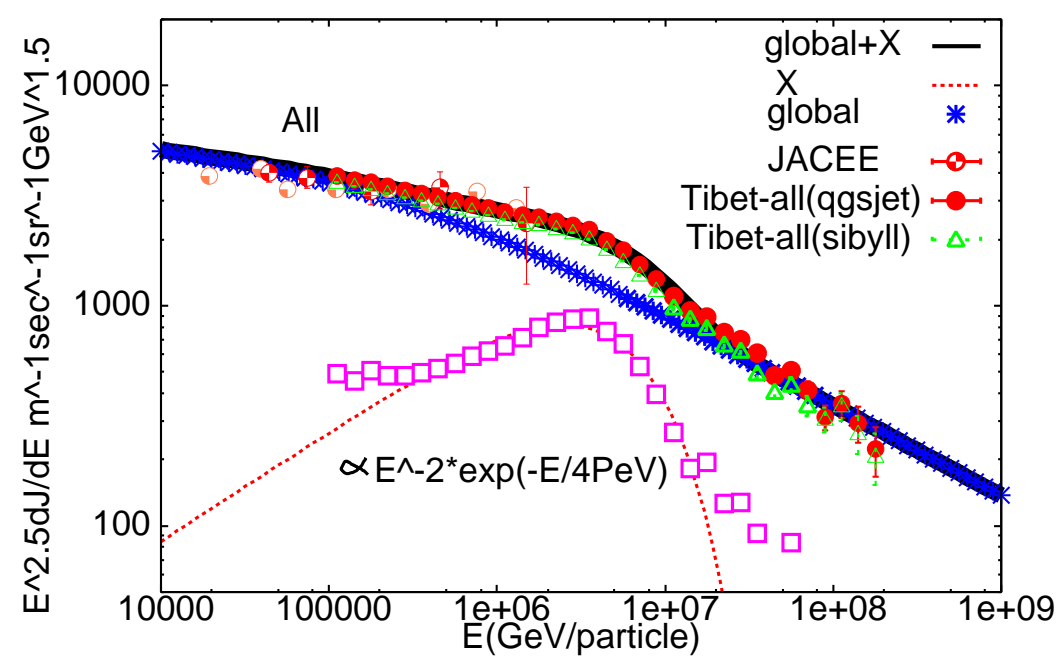

Figure 3: All-particle spectrum around the knee. Black line is the sum of the global component and an extra component whose functional form is expressed as $\propto E^{-2} \exp (-\mathrm{E} / 4 \mathrm{PeV})$.

is expected at $\mathrm{Z}$ times $100 \mathrm{TeV}$, where $\mathrm{Z}$ is the atomic number. Inspired by these theories, the following ansatz is adopted to describe the energy dependence of the flux for particles with charge Z. Here, we assume that the cut-off energy of proton $E_{c}$ is about $200 \mathrm{TeV}$. The cut-off energy for each component depends on its charge $\mathrm{Z}$, namely $E_{c}(\mathrm{z})=\mathrm{Z} * E_{c}(\mathrm{p})$ is assumed. We then sum up individual spectrum to obtain the all-particle spectrum called a global component. The numerical values of fit parameters for each chemical component are listed in Table 1. The calculated energy spectra of proton, helium, carbon, iron and the total spectrum, together with the observational data are shown in Fig. 2. The results show a good agreement with the data of each elemental species, but the sharpness of the knee is more prominent in the experimental data.

\section{Excess Component at the knee}

The all-particle spectrum above $10^{15} \mathrm{eV}$ has been obtained by many air-shower experiments, all of which have shown a sudden change in the spectral power index around $4 \times 10^{15} \mathrm{eV}$. Although our model described here has been designed to account for this change, the sharpness of the knee is more prominent in the experimental data, as shown in Fig. 2(e). Subtracting the global component from all-particle spectrum measured by the Tibet AS $\gamma$ experiment, we can see a hard spectrum of power index close to -2 , which is just the expected value of source spectrum before the modulation by the propagation, and it also shows a cutoff feature as shown in Fig. 3 indicating that the excess component is due to the contribution of nearby source(s). The enhancement around the knee can be reproduced well whose functional form is expressed as $\propto E^{-2} \exp (-\mathrm{E} / 4 \mathrm{PeV})$, as shown in Fig. 3. The sharp peak of the excess component indicates that the chemical composition is not mixed as much as global component but its main component consists of elements with limited range of atomic numbers if we adopt the rigidity dependent acceleration limit. Individual spectra of elements is calculated assuming that the excess component with certain nuclei called X component consisting entirely of proton, helium, carbon, or iron at the knee, as shown in Fig. 4. Because the experimental data points are still poor in the high energy range, it seems difficult to judge which is 

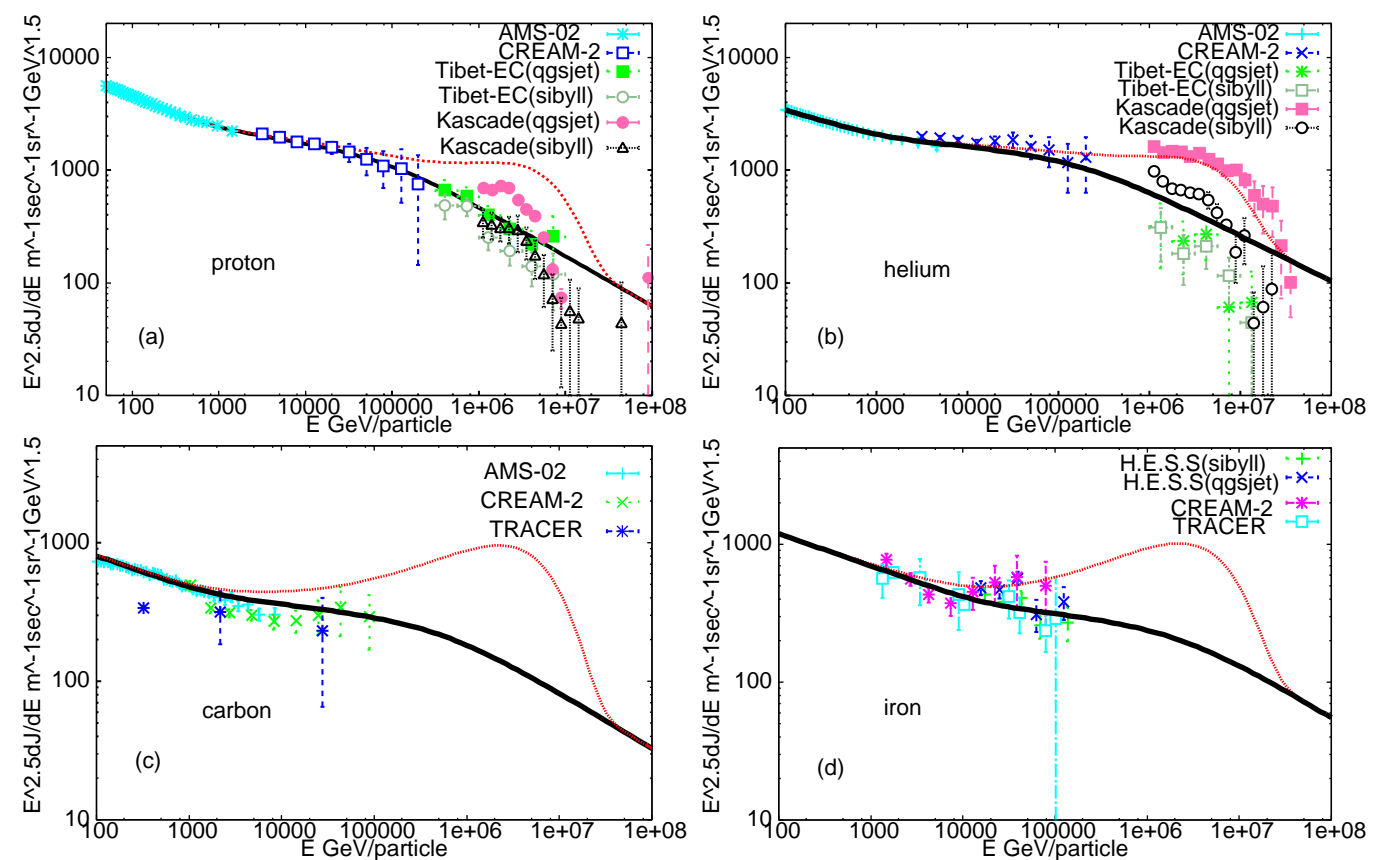

Figure 4: Individual spectra of elements is calculated assuming that the excess component with a certain nuclei called X component comprised only of proton (a), helium (b), carbon (c) and iron (d) each at the knee.

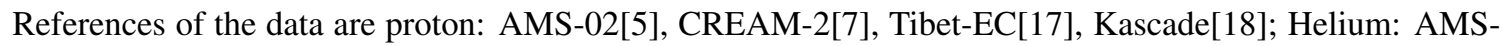

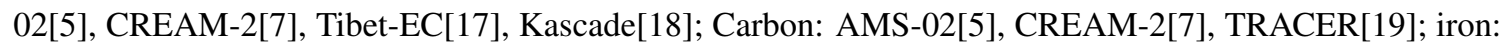

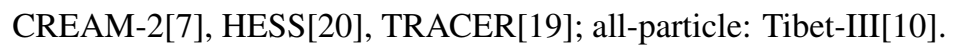

the $\mathrm{X}$ component except protons due to its much higher flux compared with present experimental data.

\section{Chemical composition around the knee}

In such a simple scenario, average mass $<\ln \mathrm{A}>$ is calculated assuming that the excess $\mathrm{X}$ component at the knee which is described in Fig.4 (a), (b), (c) and (d) as shown in Fig. 5. While the experimental data are still divergent because of the difficulty of the composition measurement at very high energy, some results fairly agree with the lines calculated by the present work. The result of Tibet-EC data for proton and helium shows that the excess component is heavier than helium. Fig. 5 may suggest that the most possible nuclei of the excess component is heavier than helium, which is not contradict with the result of Tibet-EC data[[7]].

\section{Conclusion and discussion}

We propose a phenomenological model capable of well explaining both the data by AMS-02 and others in the lower energy region and that by the Tibet AS $\gamma$ experiment simultaneously. In this model, an excess component is assumed to overlap the global component and the shape of the energy spectrum of the excess component suggests that it may be attributed to CRs from nearby source(s) since the spectral shape is very close to that expected by DSA model of SNR. This model also predicts a dominance of cosmic-ray nuclei heavier than helium at the knee. 


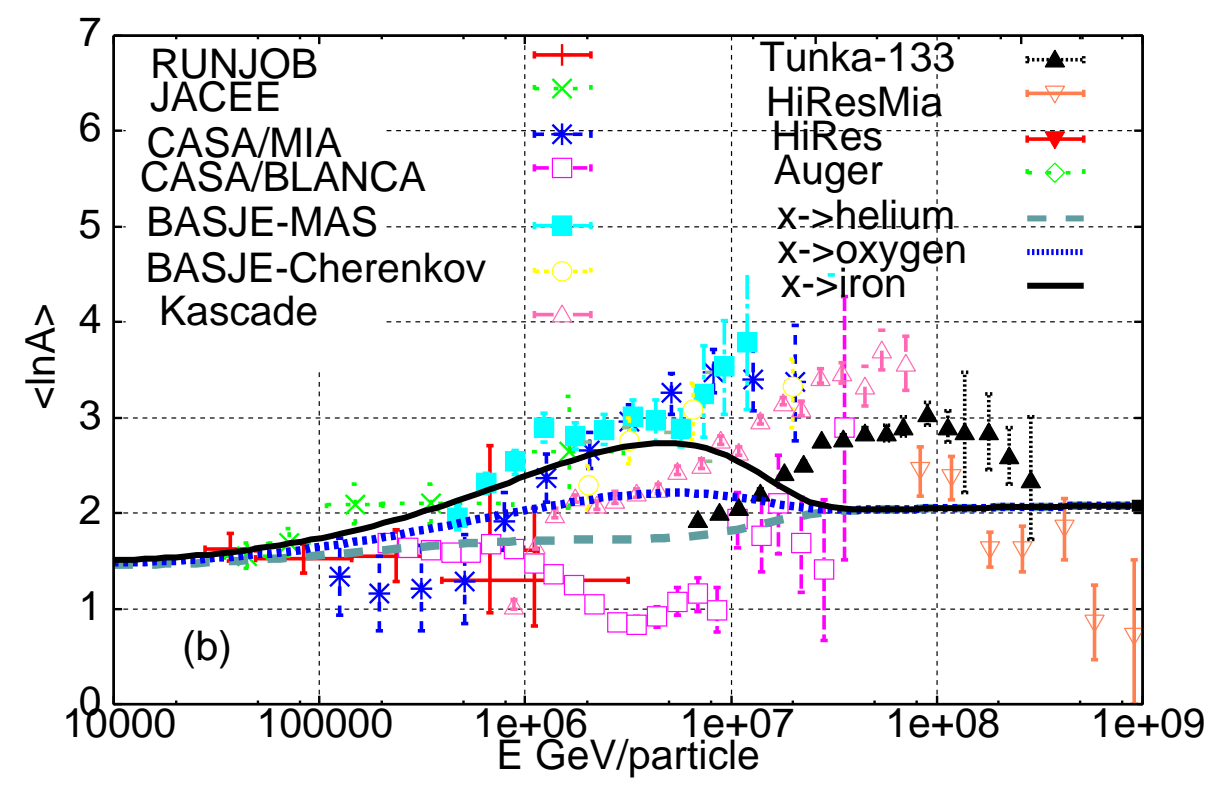

Figure 5: Average mass calculated in this model. The three lines show a case of the excess component comprised only of helium (green broken line), oxygen (blue dash line) and iron (black solid line) each.

To identify such source(s), which might be diffused, it is interesting to investigate primary gamma rays beyond $10^{13} \mathrm{eV}$, which can be produced by nuclear interactions of accelerated particles in the source region. This can be examined by observing air showers with a capability of $\mathrm{p} / \gamma$ separation. Further measurements of the chemical composition of cosmic rays will improve the model parameters used in the present analysis and enable more detailed discussion of the structure of the knee and the contribution of extra galactic cosmic rays.

\section{Acknowledgements}

The authors would like to express their thanks to the members of the Tibet AS $\gamma$ collaboration for the fruitful discussion. This work is supported by the Grants from the National Natural Science Foundation of China (11078002 and 11275212) and the Chinese Academy of Sciences (H9291450S3, 2013T2J0006) and the Key Laboratory of Particle Astrophysics, Institute of High Energy Physics, CAS. The Knowledge Innovation Fund (H95451D0U2 and H8515530U1) of IHEP, China also provide support to this study.

\section{References}

[1] Kulikov, G. V., \& Khristiansen, G. B. 1958, Soviet Physics JETP, 35, 635

[2] Haungs, A., Rebel, H., \& Roth, M. 2003, Reports on Progress in Physics, 66, 1145

[3] Abbasi, R., et al. 2005, Physics Letters B, 619, 271

[4] Abbasi, R. U., et al. 2009, Astroparticle Physics, 32, 53

[5] AMS-02 Collaboration, "AMS Days at CERNą́ and Latest Results", 15, April, 2015 Abraham, J., et al. 2010, Physics Letters B, 685, 239 
[6] For the ATIC experiment see A. D. Panovet al., Bull. Russ. Acad. Sci. Phys.73, 564 (2009)

[7] For the CREAM experiment see Y. S. Yoon et al., Astrophys. J.728, 122 (2011).

[8] For the PAMELA experiment see O. Adriani et al., Astrophys. J.765, 91 (2013); Science332, 69 (2011).

[9] See, for example, G. Bernard, T. Delahaye, P. Salati, and R. Taillet, Astron. Astrophys. 555, A48 (2013);V.S. Ptuskin, V. Zirakashvili, and E. S. Seo,Astrophys. J.763, 47 (2013); N. Tomassetti, Astrophys. J. Lett.752, L13 (2012); P. Blasi, E. Amato, and P. D. Serpico, Phys. Rev. Lett. 109, 061101 (2012); A. E. Vladimirov, G. Jíóhannesson, I. V. Moskalenko, and T. A. Porter,Astrophys. J.752, 68 (2012).

[10] Amenomori, M et al, 2008, Astrophys. J, 678, 1165-1179

[11] Berezhko, E. G., \& Ksenofontov, L. G. 1999, J. Exp. Theor. Phys., 89, 391

[12] Erlykin, A. D., \& Wolfendale, A. W. 2005, Astropart. Phys., 23, 1

[13] Ptuskin, V. S., et al. 1993, A\&A, 268, 726

[14] Wigmans, R. 2003, Astropart. Phys., 19, 379

[15] Nikolsky, S. I., \& Romachin, V. A. 2000, Phys. At. Nuclei, 63, 1799

[16] Horandel, J. R. 2003, Astropart. Phys., 19, 193

[17] Amenomori, M., et al. 2006,Phys. Lett. B,632, 58

[18] T. Antoni et al., Astropart. Phys. 24, 1 (2005).

[19] Ave, M., et al. 2008,ApJ, 678, 262

[20] Aharonian, F. A., et al. 2007,Phys.Rev.D,75, 042004 